\title{
Finite Conductance Governs the Resonance Transmission of Thin Metal Slits at Microwave Frequencies
}

\author{
J. R. Suckling, A. P. Hibbins, M. J. Lockyear, T.W. Preist, and J. R. Sambles \\ Thin Film Photonics Group, School of Physics, University of Exeter, Devon EX4 4QL, United Kingdom \\ C. R. Lawrence \\ QinetiQ, Cody Technology Park, Farnborough GU14 OLX, United Kingdom
}

(Received 18 August 2003; published 7 April 2004)

\begin{abstract}
Fabry-Perot-like resonant transmission of microwave radiation through a single subwavelength slit in a thick aluminum plate is quantified for a range of slit widths. Surprisingly, and in contrast to previous studies [e.g., Y. Takakura, Phys. Rev. Lett. 86, 5601 (2001)], the resonant frequency exhibits a maximum as a function of slit width, decreasing as the slit width is reduced to less than $2 \%$ of the incident wavelength. This result accords with a new model based on coupled surface plasmon theory taking into account the finite conductivity, and hence permittivity, of the metal. This is contrary to a common assumption that metals can be treated as infinitely conducting in this regime.
\end{abstract}

There is much current interest in the remarkable transmission of subwavelength apertures in otherwise opaque screens following the work of Ebbesen et al. [1]. While the initial study was for an array of holes in an opaque screen, further theoretical work has been undertaken for deep gratings with narrow slits in metal screens [2-6], and their Fabry-Perot-like nature has been demonstrated. The single slit case has also been studied both theoretically [7] and experimentally [8,9], and although [8] was not an exact test of [7] it indicated the correct functional form of resonant frequency on gap width.

In this Letter the resonant response of a single, deep metallic slit is studied for a range of slit widths and frequencies. It is shown that while the resonances are Fabry-Perot-like in nature they always occur at frequencies lower than that predicted from a simple Fabry-Perot model [7]. This reduction in resonant frequency with increasing slit width is anticipated simply from the different boundary conditions at the open ends of the thin slits compared to those of a Fabry-Perot interferometer. By contrast, a further reduction in resonant frequency at very narrow widths $(<70 \mu \mathrm{m})$, illustrated in the present study, is entirely associated with the finite conductivity of the aluminum. Because the resonant mode in the slit is a coupled surface mode, as the slit gap reduces so the influence of the finite skin depth in the metal becomes significant. This results in a rapidly decreasing resonant frequency and broadening resonance with reducing slit width.

Simple Fabry-Perot theory for the resonant frequencies of a cavity consisting of two nearly perfectly reflecting mirrors separated by distance $t$ is given by Eq. (1), where $c$ is the speed of light and $N$ the mode number.

$$
f=\frac{c N}{2 t} \text {. }
$$

However, a single, narrow metallic slit has different boundary conditions that cause the frequency to drop as the slit width is increased. For a perfect conductor the degree of shift in wavelength is predicted by Takakura [7] to be

$$
\frac{\lambda_{\text {shift }}}{\lambda_{\mathrm{FP}}}=\frac{2(w / t)\left[\ln \left(\pi w / \lambda_{\mathrm{FP}}\right)-3 / 2\right]}{2(w / t)\left[\ln \left(\pi w / \lambda_{\mathrm{FP}}\right)-1 / 2\right]-\pi},
$$

where $w$ is the slit width, $t$ the depth, and $\lambda_{\mathrm{FP}}$ the FabryPerot wavelength. However, for narrow slits, where the correction due to end effects may be quite small, a second, and more important effect, arises. The resonant modes, which are in effect long-range coupled surface plasmons with wave vector just beyond the light line, shift down in frequency and broaden. Sobnack et al. [10] give a formal expression for the wave vector for coupled surface plasmons in the gap between two semi-infinite, metal layers in the visible regime. For the microwave domain, when the metal permittivity is dominated by the imaginary component, their expression is found [11] simply to reduce to

$$
f=f_{\mathrm{FP}}-\frac{c}{2 \sqrt{2}\left|\varepsilon_{i}\right|^{1 / 2} w \pi},
$$

where $f_{\mathrm{FP}}$ is the simple Fabry-Perot frequency of the cavity, $w$ is the width of the slit, and $\varepsilon_{i}$ is the imaginary part of the permittivity of the metal. A slit of infinitesimal width in a perfect conductor is predicted to have a resonant frequency equal to the Fabry-Perot cavity of the same depth [7]. By complete contrast Eq. (3) predicts a turn down in the resonant frequency as the slit approaches zero width, with the resonant frequency going to zero (wavelength to infinity) if the metal has finite conductivity. 
The microwave regime was chosen for this experiment for two main reasons. First, the available analytic theory for single slit response [7] assumes that the metal is perfectly conducting. While this is certainly not true at optical frequencies, it has been taken by many to be a good assumption in the microwave regime. However, our results forcefully demonstrate that this is not correct. Second, it is clearly far easier to manufacture the structure and alter its parameters in the microwave regime than in the visible.

The experimental setup is shown in the inset of Fig. 1. The slit is made up of two large, polished aluminum plates of face area approximately $400 \times 200 \mathrm{~mm}^{2}$ and thickness $19.58 \mathrm{~mm}$. The machining of the plates is to such an accuracy that the slit width varies by less than $25 \mu \mathrm{m}$. The plates are separated by smooth, dielectric spacers placed outside the incident beam spot, and the transmission through the structure for a range of slit widths $(15 \leq w \leq 1000 \mu \mathrm{m})$ is characterized. The slit is placed directly between two microwave horns, one acting as source and the other as detector. The detector horns are placed at $\sim 10 \mathrm{~cm}$, far enough away not to be considered near field, yet as close as possible to give acceptable signals from the very narrow slits. A typical set of data is shown in Fig. 1 for a $250 \mu \mathrm{m}$ slit width.

An infinite plane wave source induces no momentum along the slit length (for normally incident radiation); however, experimentally this is impossible to attain. The momentum associated with the Fourier transform of a finite-width beam width results in a corresponding increase in resonant frequency. It is essential that this is well quantified to allow very accurate determination of the influence of slit width on the resonant frequencies to be extracted. To characterize this frequency shift effect the slit length was constrained with parallel aluminum spacers, replacing the dielectric, and the change in reso-

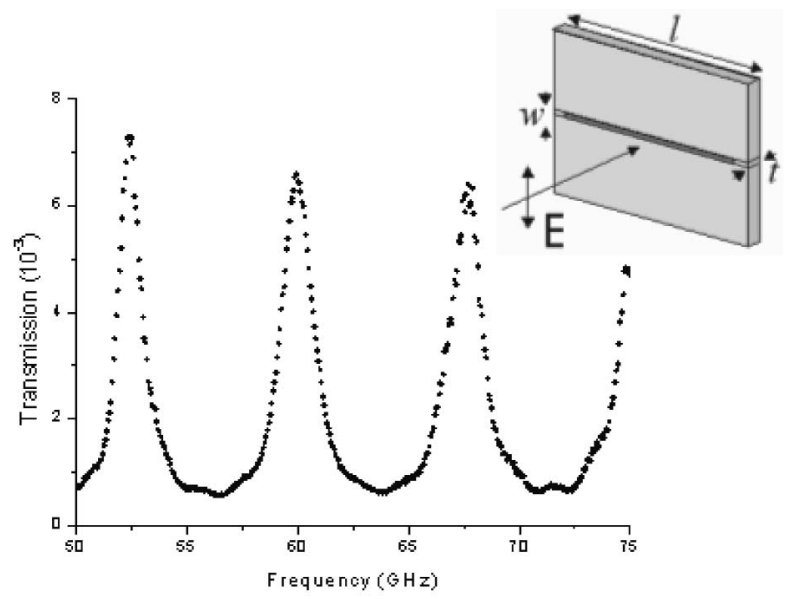

FIG. 1. Resonant transmission of a $250 \mu \mathrm{m}$ slit. Inset: the slit labeled with dimensions and polarization of incident microwaves. nant frequency as a function of slit length characterized. This change in frequency is given, to first order, by

$$
\Delta f \propto \frac{1}{f_{\mathrm{FP}} l^{2}},
$$

where $l$ is the slit length. As expected from this simple theory, a graph of frequency against inverse slit length squared gave a straight line (see inset of Fig. 2). The intersection of this line with the frequency axis gives the "infinite" beam resonant frequency, and comparing this with the frequency actually measured for the sample of infinite slit length gave the frequency shift due to the finite beam width. These shifts, which were subtracted from all the experimental data to allow comparison with
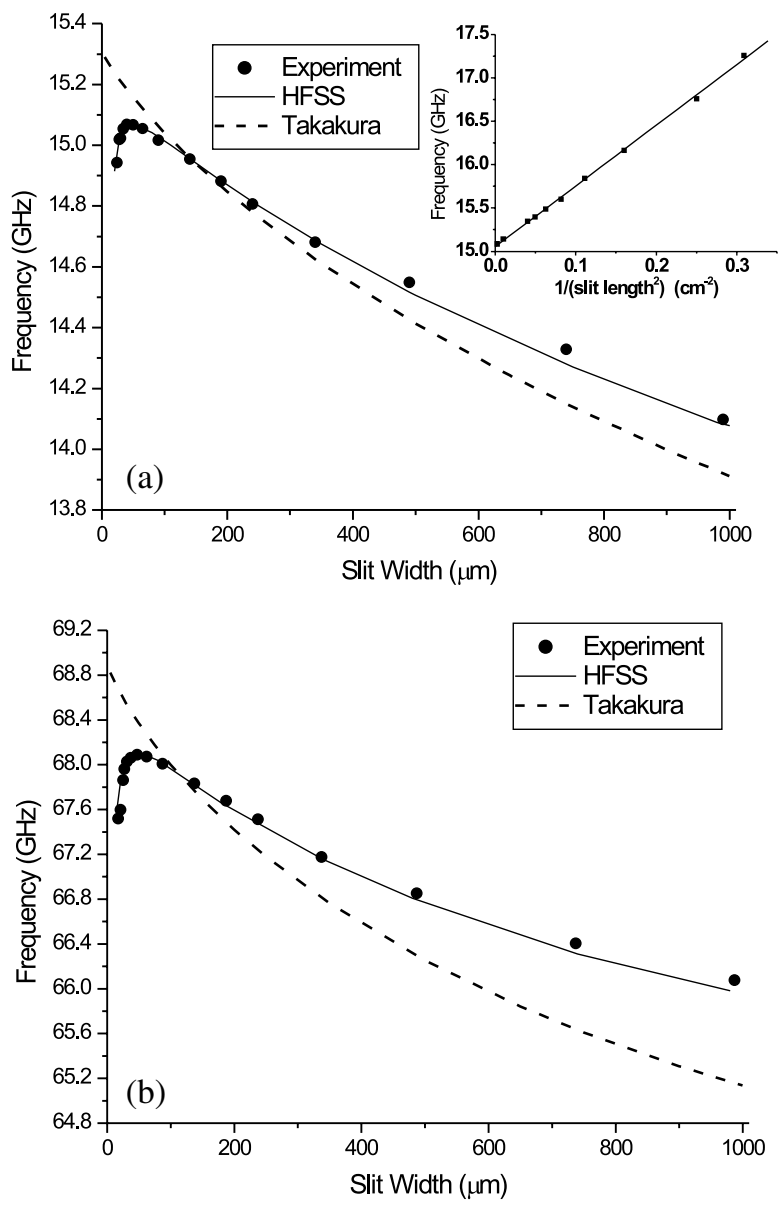

FIG. 2. (a) Mode 2: Experimental data, slit widths 20-990 $\mu \mathrm{m}$ (points), resonant frequency corrected by $-0.019 \pm 0.005 \mathrm{GHz}$ due to finite beam spot size, width corrected by $-10 \pm 4 \mu \mathrm{m}$ due to plate bowing and surface roughness. HFSS [12] modeling (solid line) and Takakura prediction (dashed line). Inset: frequency against inverse slit length squared for slit width of $80 \mu \mathrm{m}$ (points), linear fit (solid line), frequency at intercept $\sim 15.05 \mathrm{GHz}$. (b) Mode 9: Experimental data for slit widths 24-994 $\mu \mathrm{m}$ (points), resonant frequency corrected by $-0.035 \pm 0.008 \mathrm{GHz}$, width corrected by $-13 \pm$ $3 \mu \mathrm{m}$, HFSS modeling (solid line) and Takakura prediction (dashed line). 
plane wave modeling, were found to be $\sim 0.035 \mathrm{GHz}$ for the $N=9$ mode at $68.6 \mathrm{GHz}$, corresponding to a beam width of $\sim 68 \mathrm{~mm}$ diameter, and $\sim 0.019 \mathrm{GHz}$ for the $N=2$ mode at $15.1 \mathrm{GHz}$, corresponding to a beam width of $\sim 190 \mathrm{~mm}$. In addition, the plates, separated by spacers at the ends, bowed slightly to close the slit, requiring a correction of slit width when fitting to theory, stated in captions to Figs. 2(a) and 2(b).

(Note also from Fig. 1 that the peak transmission is of order $8 \times 10^{-3}$ for a $\lambda: w$ ratio of about $20: 1$. For similar ratios Takakura [7] predicts a transmission of $2.5 \times 10^{-3}$. This is in reasonable agreement considering the strong diffraction of the microwaves at the slit exit.)

Figure 2 shows the frequency dependence of two modes as a function of slit width for the $N=2$ mode $(\sim 15.1 \mathrm{GHz})$ and the $N=9$ mode $(\sim 68.1 \mathrm{GHz})$. Also shown for comparison is the Takakura [7] prediction and that from a finite element computer model [12]. While Takakura's prediction has the correct form, starting at the Fabry-Perot frequency and dropping monotonically in frequency with increasing slit width, it is clear that the magnitude does not accord with the data. The finite element model with finite conductivity agrees very well with the data.

The turn down in resonant frequency for the very narrow slits confirms the Preist-Sambles model [Eq. (3)] for coupled surface plasmons at microwave frequencies. This drop in frequency with decreasing slit width also provides a method for determining the imaginary permittivity of aluminum. To generate the predictions that compare with the data the computer modeling code uses finite conductivity in the aluminum. From this it generates the relative imaginary permittivity using [13]

$$
\varepsilon_{i}=\frac{g}{2 \pi \varepsilon_{0} f},
$$

where $g$ is the conductivity and $f$ the frequency of incident radiation. This equation may be applied when the photon frequency (here $\sim 10^{9} \mathrm{~s}^{-1}$ ) is much less than the damping constant of the metal, $\gamma \approx 10^{14} \mathrm{~s}^{-1}$. In the microwave regime the conductivity of the metal would be expected to be close to that of the d.c. value for aluminum, $3.8 \times 10^{7} \mathrm{~S} \mathrm{~m}^{-1}$. The conductivities required to fit the data, $3.5 \pm 0.3 \times 10^{7} \mathrm{Sm}^{-1}$ and $1.7 \pm 0.1 \times$ $10^{7} \mathrm{~S} \mathrm{~m}^{-1}$ for modes $N=2$ and 9, respectively, give imaginary permittivity values of $4.17( \pm 0.35) \times 10^{7}$ at $15.1 \mathrm{GHz}$ and $4.48( \pm 0.26) \times 10^{6}$ at $68.1 \mathrm{GHz}$, respectively. Although the conductivity values are close to that expected, their variance suggests that the simple Drude model may not be appropriate. It is worth noting that as the conductivity is reduced, for any given mode, the frequency turn down begins at increasingly wider slits, as also predicted by Preist-Sambles [Eq. (3)].

Figure 3 shows the fall in resonant frequency from a fixed slit width $(500 \mu \mathrm{m})$ across the full range of frequencies measured $(\sim 15-69 \mathrm{GHz})$ compared to the simple

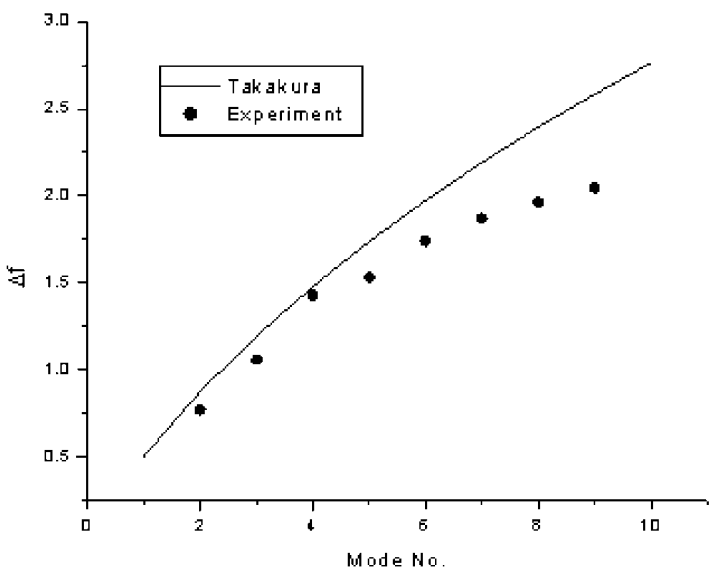

FIG. 3. Mode shifts from the simple Fabry-Perot model, as given by Takakura predictions and the data for a $500 \mu \mathrm{m}$ slit.

Fabry-Perot prediction for the same depth interferometer. Also plotted are Takakura's [7] predictions. Once again, it is clear that [7] has the right form, but predicts too low a frequency for each mode. This trend shows that it is the ratio of the wavelength to the slit width, and not the number of wavelengths inside the slit, that determines the shift in frequency away from that of the simple FabryPerot prediction. It might be expected that more modes within the slit would minimize boundary effects, instead, as the frequency increases, the slit width becomes a more significant fraction of the wavelength, and causes a greater shift in frequency.

In summary, the frequency of resonant transmission of a narrow metallic slit has been measured as a function of slit width. It is shown that one approximate theory [7] is flawed but that the data are in accordance with predictions of a finite element code when finite conductivity is also taken into account. This is in contradiction to the assumption that metals can be treated as perfect conductors in the microwave regime. It is also shown that for small slit widths a simple coupled surface plasmon model predicts the observed reduction in resonant frequency and from fitting to this gap-dependent change in frequency the imaginary relative permittivity of aluminum at microwave frequencies has also been determined. As expected, the value of the imaginary permittivity was found to change by almost one order of magnitude across the range of frequencies measured.

The authors thank the Engineering and Physical Sciences Research Council and QinetiQ (Farnborough) for their financial support.

[1] T.W. Ebbesen, H. J. Lezec, H. F. Ghaemi, T. Thio, and P. A. Wolff, Nature (London) 391, 667 (1998).

[2] J. A. Porto, F. J. Garcia-Vidal, and J. B. Pendry, Phys. Rev. Lett. 83, 2845 (1999). 
[3] S. Astilean, Ph. Lalanne, and M. Palamaru, Opt. Commun. 175, 265 (2000).

[4] S. Collin, F. Pardo, T. Teissier, and J-L. Pelouard, J. Opt. A, Pure Appl. Opt. 4, 154 (2002).

[5] H. E. Went, A. P. Hibbins, J. R. Sambles, C. R. Lawrence, and A. P. Crick, Appl. Phys. Lett. 77, 2789 (2000).

[6] A. P. Hibbins, J. R. Sambles, C. R. Lawrence, and D. M. Robinson, Appl. Phys. Lett. 79, 2844 (2001).

[7] Y. Takakura, Phys. Rev. Lett. 86, 5601 (2001).

[8] F. Yang and J. R.Sambles, Phys. Rev. Lett. 89, 063901 (2002).
[9] A. P. Hibbins, J. R. Sambles, and C. R. Lawrence, Appl. Phys. Lett. 81, 4661 (2002).

[10] M. B. Sobnack, W.C. Tan, N.P. Wanstall, T.W. Preist, and J. R. Sambles, Phys. Rev. Lett. 80, 5667 (1998).

[11] T.W. Preist and J. R. Sambles (private communication).

[12] HFSS, Ansoft Corporation, Pittsburgh, PA, U.S.A.

[13] J. R. Reitz, F. J. Milford, and R.W. Christy, Foundations of Electromagnetic Theory (Addison-Wesley, Wokingham, 1993), 4th ed. 